\title{
Vinyl Ketone Reagents for Covalent Protein Modification. Nitroxide Derivatives Suited to Rotational Diffusion Studies by Saturation Transfer Electron Spin Resonance, Using Membrane-Bound $\mathrm{Na}, \mathrm{K}-\mathrm{ATP}$ ase as an Example
}

\author{
Mikael Esmann,* H. Olga Hankovszky, † Kálmán Hideg, † Jens Arne Pedersen, $\ddagger$ and Derek Marsh§ \\ *Institute of Biophysics, University of Aarhus, Aarhus, Denmark; †Central Laboratory for Chemistry, University of Pécs, \\ Pécs, Hungary; $\ddagger$ Institute of Chemistry, University of Aarhus, Aarhus, Denmark; and §Max-Planck-Institut \\ für Biophysikalische Chemie, Abteilung Spektroskopie, Göttingen, Federal Republic of Germany
}

Received February 23, 1990

The reactivity of a series of substituted vinyl ketone nitroxides with an integral membrane protein, the Na,K-ATPase, is described. Increasing the electrophilicity of the conjugated double bond enhances reactivity markedly, with some spin labels showing higher reactivity than the conventionally used maleimide derivatives. The spectroscopic characteristics of the spinlabeled protein are also better suited for motional analysis by the saturation transfer electron spin resonance (STESR) method than with previous labeling procedures. The rotational correlation time, deduced from STESR experiments, is in the same range (100-300 $\mu \mathrm{s})$ irrespective of the vinyl ketone derivative used, and the rotational mobility corresponds to an $(\alpha \beta)_{2}$ or higher oligomer of the membrane-bound Na,K-ATPase. 1990 Academic Press. Inc.

The covalent modification of nucleophilic amino acid residues using electrophilic reagents is a generally useful technique in protein chemistry, e.g., in investigating structure-function relationships. Reagents such as maleimide, iodoacetic acid, and iodoacetamide are used extensively for the labeling of protein $\mathrm{SH}$ groups (1). Analogues of these reagents bearing a spectroscopic reporter group can be used as environmentally sensitive probes to study protein structure and dynamics. The important features of such covalent probes are the reactivity and mode of attachment to the protein residues. The latter property determines the sensitivity of the probe to overall protein rotation as well as to protein segmental motion and conformation.
Covalent reagents bearing the nitroxide free radical moiety (spin labels) in conjunction with saturation transfer electron spin resonance (STESR) ${ }^{1}$ spectroscopy have been found to be particularly useful for the study of protein motions in the microsecond time scale $(2,3)$. Examples of the latter are the rotational diffusion of integral proteins in membranes, the formation of supramolecular protein aggregates, and large-scale segmental motions in proteins.

\footnotetext{
${ }^{1}$ Abbreviations used: 5-MSL, 3-maleimido-1-oxyl-2,2,5,5-tetramethylpyrrolidine; 5-InVSL, 2-[(1-oxyl-2,2,5,5-tetramethy]-3pyrrolin-3-yl)methenyl]indane-1,3-dione; 5-BzVSL, 1-oxyl-2,2,5,5tetramethyl-3-(2-benzoylethenyl)-3-pyrroline; 5-DiBzVSL, 1 oxyl-2,2,5,5-tetramethyl-3-(2,2-dibenzoylethenyl)-3-pyrroline; 5HBzVSL, 1-oxyl-2,2,5,5-tetramethy]-3-[2-(4'-hydroxybenzoyl)ethenyl]pyrroline; 5-CBzVSL, 1-oxyl-2,2,5,5-tetramethyl-3-[2-(4'carboxybenzoyl)ethenyl]pyrroline; 5-HNBzVSL, 1-oxyl-2,2,5,5tetramethyl-3-[2-(3'-nitro-4'-hydroxybenzoyl)ethenyl $]$ pyrroline; 5 HDiNBzVSL, 1-oxyl-2,2,5,5-tetramethyl-3-[2-(3',5'-dinitro-4'-hydroxybenzoyl)ethenyl]-3-pyrroline; 5-(2)-PyVKSL, 1-oxyl-2,2,5,5tetramethyl-3-[2-(2'-pyridylcarbonyl)ethenyl]pyrrolin-3-yl tosylate; 6-InVSL, 2-[(1-oxyl-2,2,6,6-tetramethy]-1,2,5,6-tetrahydropyridin-4yl)methenyl]indane-1,3-dione; 5-(2)-CBziVSL, 2-[(3-(1-oxy]-2,2, 5,5-tetramethyl-3-pyrrolin-3-yl)etheno)carbonyl]benzimidazole; 5(3)-IndVSL, 3-[(3-(1-oxyl-2,2,5,5-tetramethyl-3-pyrrolin-3-yl)etheno)carbonyl]indole; 5-DiCVVSL, 1-oxyl-2,2,5,5-tetramethyl-3pyrrolin-3-methenylmelodinitrile; 5-EtCxVSL, ethyl 2-cyano-3-(1oxyl-2,2,5,5-tetramethyl-3-pyrrolin-3-yl)acrylate; 5-MeldVSL, 5-[(1oxyl-2,2,5,5-tetramethyl - 3-pyrrolin - 3-yl)methenyl] - 2,2 - dimethyl4,6-dioxo-1,3-dioxane; PyMIn, 2-(4-pyridyl)methenylindane-1,3dione; PyMInMI, 2-(4-pyridyl)methenylindane-1,3-dione methiodide; NEM, $N$-ethylmaleimide; CDTA, trans-1,2-diaminocyclohexane- $N, N, N^{\prime}, N^{\prime}$-tetraacetic acid; EWG, electron-withdrawing-group; STESR, saturation transfer ESR; $V_{1}$, first harmonic ESR absorption signal detected in-phase with respect to the field modulation; $V_{2}^{\prime}$, second harmonic absorption signal detected $90^{\circ}$ out-of-phase with respect to the field modulation; $\mathrm{SH}$ group, cysteine residue.
} 
The present paper deals with a new class of covalent reagents based on the reactive vinyl ketone--or similar-moieties. These reagents react by a Michael addition across the double bond, in a manner similar to maleimide (4):

$$
\mathrm{R}-\mathrm{SH}+\mathrm{R}^{\prime} \mathrm{CH}=\mathrm{CHCOR}{ }^{1} \rightarrow \mathrm{R}^{\prime} \mathrm{CH}(\mathrm{RS}) \mathrm{CH}_{2} \mathrm{COR}^{1} .
$$

Here the $R^{\prime}$ radical may bear a reporter group, e.g., a nitroxide spin label. Sulfhydryl addition is likely to take place at the $\beta$-position of the vinyl bond and, by suitable design, the length of linkage to the reporter-bearing group may be optimized. The reactivity of the vinyl ketone center is determined partly by steric effects, but most importantly by the electron-withdrawing groups (EWGs) attached at the $\mathrm{R}^{1}$ radical.

The $\mathrm{Na}, \mathrm{K}$-ATPase is a large integral membrane transport enzyme which contains 24 cysteine residues per $112 \mathrm{kD}$ in the primary sequence (in both kidney and torpedo enzyme; Refs. $(5,6))$. Approximately six of these $\mathrm{SH}$ groups may be labeled by $N$-ethylmaleimide when the protein is in its nondenatured state. Operationally, the accessible cysteines may be divided into two groups (7). Class I groups can be labeled in the presence of $40 \%$ glycerol without affecting the enzyme activity. The remaining Class II groups can normally only be labeled in the absence of glycerol leading to inhibition of the overall activity. Under suitable conditions the ability of the enzyme to be phosphorylated by ATP may be retained (8).

For the present studies, we have used the membranous $\mathrm{Na}, \mathrm{K}$-ATPase to screen a series of vinyl ketones and similar reagents, both for reactivity and for the spectroscopic properties of spin-labeled derivatives. In the latter case, the principal object has been to find labels having high reactivity but displaying little sensitivity to segmental motion, hence being ideally suitable for the study of overall protein rotational diffusion using STESR. Previously, using the benzyl vinyl ketone derivative, we have been able to demonstrate the potential improvement relative to the conventional maleimide spin label for such studies (9). However, the benzyl derivative was found to have lower solubility and considerably reduced reactivity compared to that of the maleimide labels.

Now we have synthesized 15 further reagents with different polarities and stronger EWGs. The indanedione spin-labeled derivative and the non-spin-labeled 2-(4-pyridyl)methenylindane-1,3-dione and its methiodide salt were found to be very highly reactive, even more so than maleimide. These compounds are therefore likely to be generally useful as protein modification reagents, particularly in spin label STESR studies. All the spin-labeled reagents investigated monitor rotational motion in the same time range, even though their second-order rate constants for inactivation of

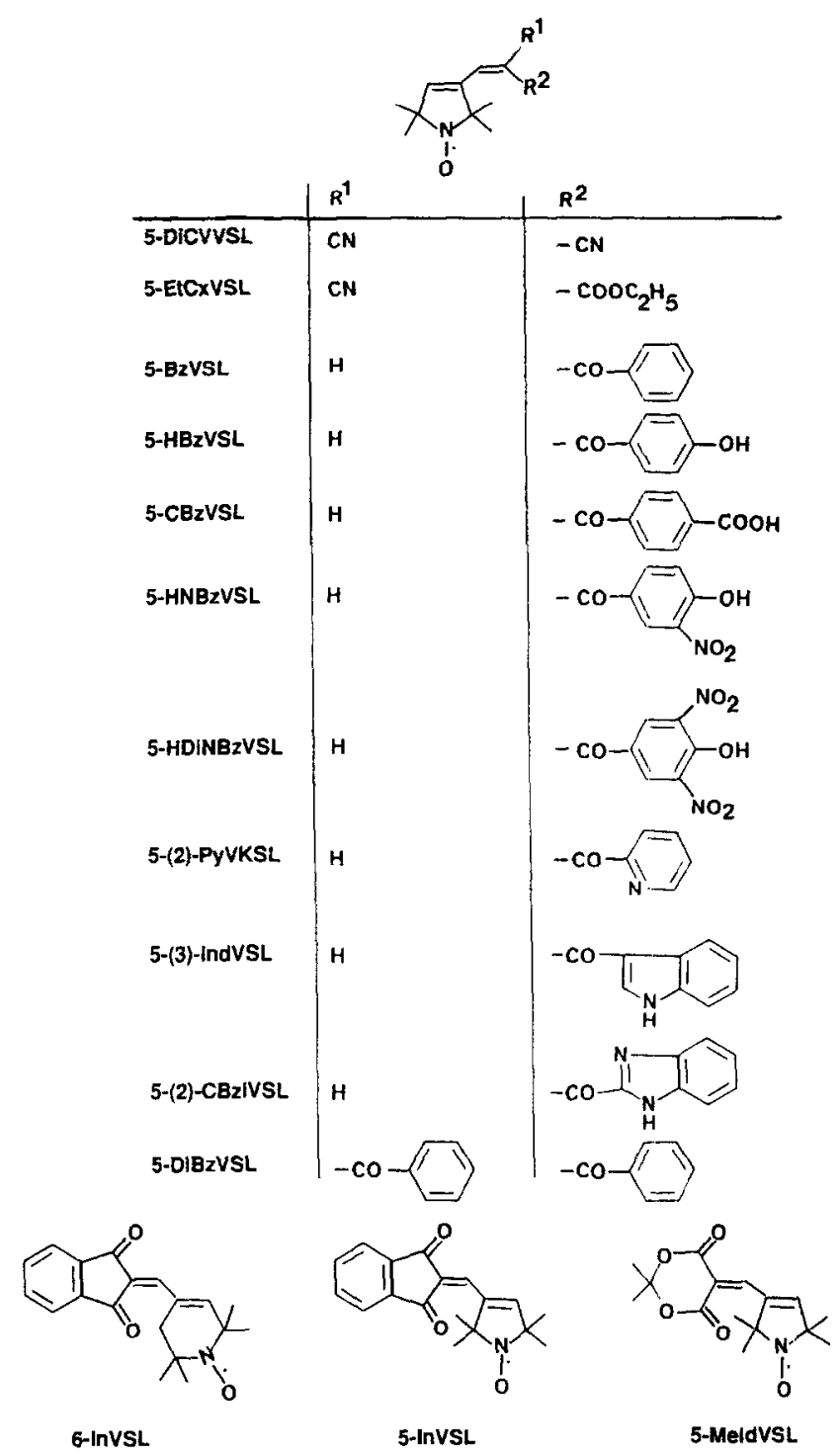

FIG. 1. Structures of sulfhydryl reagents used in this study.

$\mathrm{Na}, \mathrm{K}-\mathrm{ATPase}$ vary by a factor of 200 . The availability of a range of reagents with different reactivities may also offer the potential for experiments involving more selective labeling.

\section{MATERIALS AND METHODS}

Spin Label Preparation

Spin labels (see Fig. 1) were synthesized according to Hankovszky et al. (10). The general reaction for synthesis of the various nitroxides was a condensation of a nitroxide aldehyde with appropriate compounds containing an active methylene group, giving $\alpha, \beta, \gamma, \delta$-dienes (cf. Ref. (10) and Fig. 1). It has been shown previously that these reagents add to $\mathrm{SH}$ groups at the $\beta$-carbon atom of the diene (10).

Synthesis of 2-(4-pyridyl)methenylindane-1,3-dione. The compound was prepared by the same method as 
described for spin-labeled dienones (10): A solution of 4-pyridinecarboxaldehyde ( $3.0 \mathrm{mmol}, 321 \mathrm{mg})$ and $1,3-$ indanedione $(3.0 \mathrm{mmol}, 438 \mathrm{mg})$ in dry benzene $(50 \mathrm{ml})$ was heated to reflux in the presence of catalysts/benzoic acid $(10 \mathrm{mg})$ and piperidine $(10 \mathrm{mg})$ in a Dean-Stark apparatus for $6 \mathrm{~h}$, during which time water was collected.

The mixture was cooled at room temperature and washed with $\mathrm{NaHCO}_{3}$ and brine. The benzene phase was dried $\left(\mathrm{MgSO}_{4}\right)$ and flash chromatographed on silica gel to give an orange-yellow crystalline product $366 \mathrm{mg}$ (52\%), $\mathrm{mp} 169-170^{\circ} \mathrm{C}$.

Analytical values for PyMIN $\left(\mathrm{C}_{15} \mathrm{H}_{9} \mathrm{NO}_{2} ; \mathrm{MW} 235.3\right)$. Calcd: C, 76.59; H, 3.86; N, 5.95\%. Found: C, 76.60; H, $4.01 ; \mathrm{N}, 5.89 \%$.

Synthesis of 2-(4-pyridyl)methenylindane-1,3-dione methiodide. To the solution of the above base $(1.0$ $\mathrm{mmol}, 235 \mathrm{mg})$ in dry acetone $(10 \mathrm{ml})$ was added iodomethane $(2.0 \mathrm{mmol}, 284 \mathrm{mg}$ ) and the resulting mixture refluxed for $30 \mathrm{~min}$. The separated red crystals were filtered to give the methiodide salt $339 \mathrm{mg}(90 \%), \mathrm{mp}$ $>265^{\circ} \mathrm{C}$.

Analytical values for PyMInMI $\left(\mathrm{C}_{16} \mathrm{H}_{12} \mathrm{INO}_{2} ; \mathrm{MW}\right.$ 377.2). Calcd: C, 50.95; H, 3.21; N, 3.71\%. Found: C, $50.86 ; \mathrm{H}, 3.12 ; \mathrm{N}, 3.83 \%$.

\section{Enzyme Preparations and Labeling}

$\mathrm{Na}, \mathrm{K}-\mathrm{ATP}$ ase from the rectal gland of Squalus acanthias was prepared as described by Skou and Esmann (11), but without the treatment with saponin. The $\mathrm{Na}, \mathrm{K}$-ATPase typically constituted $50-70 \%$ of the protein (determined as the content of $\alpha$ - and $\beta$-subunits from SDS gel electrophoresis), and the specific activity ranged accordingly from 1100 to $1500 \mu \mathrm{mol}$ ATP hydrolyzed/mg protein per hour. Na,K-ATPase activity and protein content were determined as previously described (12).

Prelabeling of Na,K-ATPase with NFM to block Class I SH groups and sulfhydryl groups of non- $\mathrm{Na}, \mathrm{K}$ ATPase proteins in the membrane preparations was performed as follows (see Ref. (13) for details): $\mathrm{Na}, \mathrm{K}$-ATPase (approx $1 \mathrm{mg} / \mathrm{ml}$ ) was incubated at $23^{\circ} \mathrm{C}$ with $0.1 \mathrm{mM}$ NEM in $30 \mathrm{mM}$ histidine $\left(\mathrm{pH} 7.0\right.$ at $\left.23^{\circ} \mathrm{C}\right) / 5$ $\mathrm{mM}$ CDTA $/ 150 \mathrm{mM} \mathrm{KCl}$ and $36 \%$ (v/v) glycerol for 60 $\mathrm{min}$. The reaction was stopped by addition of $1 \mathrm{mM} 2$ mercaptoethanol, and the membranes were washed by centrifugation in $20 \mathrm{mM}$ histidine $\left(\mathrm{pH} 7.0\right.$ at $\left.20^{\circ} \mathrm{C}\right)$ and $25 \%(\mathrm{v} / \mathrm{v})$ glycerol at $200,000 \mathrm{~g}$. Three centrifugations in 27-ml tubes were sufficient to remove residual reaction medium. The prelabeled enzyme was stored in $20 \mathrm{mM}$ histidine and $25 \%(\mathrm{v} / \mathrm{v})$ glycerol at $-20^{\circ} \mathrm{C}$.

Selective spin-labeling of the Class II SH groups, which are essential for the overall $\mathrm{Na}, \mathrm{K}$-ATPase activity, was done as follows (13): Prelabeled Na,K-ATPase (see above) was incubated with the required amount of nitroxide-labeled reagent at $37^{\circ} \mathrm{C}$ in $30 \mathrm{~mm}$ histidine $\left(\mathrm{pH} 7.4\right.$ at $37^{\circ} \mathrm{C}$ ) in the presence of $150 \mathrm{mM} \mathrm{KCl} / 5 \mathrm{mM}$ CD'TA/3 mM ATP (Tris salt). The reaction was stopped by addition of $1 \mathrm{mM} \mathrm{2-mercaptoethanol,} \mathrm{and} \mathrm{the} \mathrm{mem-}$ branes were washed by centrifugation in $20 \mathrm{mM}$ histidine $\left(\mathrm{pH} 7.0\right.$ at $\left.20^{\circ} \mathrm{C}\right)$ and $25 \%(\mathrm{v} / \mathrm{v})$ glycerol at $200,000 \mathrm{~g}$. The spin-labeled enzyme was stored in $20 \mathrm{mM}$ histidine and $25 \%(\mathrm{v} / \mathrm{v})$ glycerol at $-20^{\circ} \mathrm{C}$. The various spin labels were added as dimethylformamide (DMF) or ethanol solutions. The final DMF or ethanol concentration was less than $1 \%$. This concentration of organic solvent in the incubation medium had no effect on enzyme activity.

\section{ESR Spectroscopy}

Samples for ESR spectroscopy were prepared according to following protocol (14): $1 \mathrm{mg}$ of spin-labeled protein was diluted in $10 \mathrm{ml}$ buffer ( $30 \mathrm{~mm}$ histidine $(\mathrm{pH} 7.4$ at $\left.37^{\circ} \mathrm{C}\right) / 100 \mathrm{mM} \mathrm{NaCl} / 1 \mathrm{mM}$ CDTA) and the membranes pelleted by centrifugation at $6^{\circ} \mathrm{C}$ for $45 \mathrm{~min}$ at $100,000 \mathrm{~g}$. The pellet was freed from excess buffer, taken up into a $1 \mathrm{~mm}$ diameter glass capillary, and trimmed to a sample length of $5 \mathrm{~mm}$.

ESR spectra were recorded on a Varian E-12 Century Line $9-\mathrm{GHz}$ spectrometer equipped with nitrogen gas flow temperature regulation. Conventional, in-phase, absorption ESR spectra ( $V_{1}$ display) were recorded with a modulation frequency of $100 \mathrm{kHz}$ and a modulation amplitude of $1.6 \mathrm{G}$ peak-to-peak, at the same microwave power as used for recording the STESR spectra. STESR spectra were recorded in the second harmonic, $90^{\circ}$ out-of-phase, absorption mode ( $V_{2}^{\prime}$ display) with a modulation frequency of $50 \mathrm{kHz}$ and a modulation amplitude of $5 \mathrm{G}$ peak-to-peak. Standardized sample geometry and spectrometer settings and calibrations were employed as in the protocol described in Refs. $(15,16)$. Integrals of the STESR spectra, normalized with respect to the intensity of the $V_{1}$-mode spectra, were evaluated as described in Ref. (17). Calibrations of the diagnostic STESR lineheight ratios $\left(L^{\prime \prime} / L\right.$ and $\left.H^{\prime \prime} / H\right)$ and normalized integral intensities, in terms of the rotational correlation times of 5-MSL-labeled hemoglobin, were taken from Ref. (23). Further details of the ESR spectroscopy are given in Ref. (14).

\section{RESULTS}

Na,K-ATPase Class II SH groups were spin-labeled at $37^{\circ} \mathrm{C}$ after the Class I SH groups had been prelabeled with NEM. Labeling of Class II SH groups in the presence of $150 \mathrm{mM} \mathrm{K}^{+}$and $3 \mathrm{mM}$ ATP yields inactivation of overall $\mathrm{Na}, \mathrm{K}$-ATPase activity, but the ability to phosphorylate the enzyme is retained (8).

The time course of inactivation of Na,K-ATPase on reaction with the vinyl ketone 5-(2)-CBziVSL is given in Fig. 2A. The inactivation is biphasic on a semilog plot 

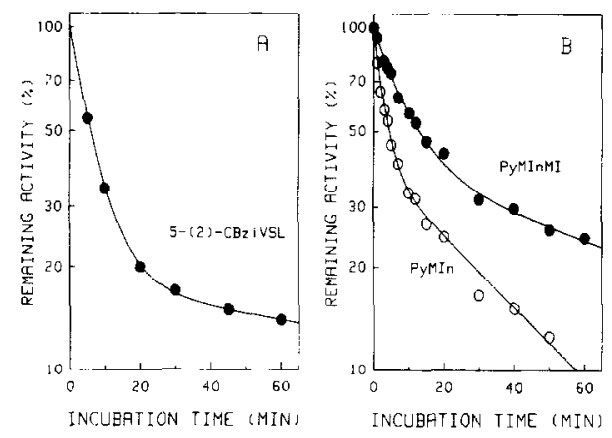

FIG. 2. Time course of inactivation of $\mathrm{Na}, \mathrm{K}-\mathrm{ATPase}$ (A) Inactivation by 5-(2)-CBziVSL. Na,K-ATPase membranes $(1 \mathrm{mg} / \mathrm{ml})$ were incubated at $37^{\circ} \mathrm{C}$ in the presence of $30 \mathrm{mM}$ histidine, $5 \mathrm{mM}$ CDTA, $150 \mathrm{mM} \mathrm{KCl}$, and $0.32 \mathrm{mM} 5$-(2)-CBziVSL. The residual activity is given in percent of the activity at $t=0$ on a logarithmic scale. The curved line represents a sum of two exponentials of the form $A(t)$ $=82 \cdot \exp (-0.154 \cdot t)+18 \cdot \exp (-0.0042 \cdot t)$, yielding second-order rate constants for the two phases of inactivation of 0.48 and 0.013 $\mathrm{mM}^{-1} \cdot \min ^{-1}$, respectively. (B) Inactivation by PyMIn (open circles) and PyMInMI (filled circles). Na,K-ATPase membranes $(1 \mathrm{mg} / \mathrm{ml}$ ) were incubated at $37^{\circ} \mathrm{C}$ in the presence of $30 \mathrm{~mm}$ histidine, $5 \mathrm{mM}$ CDTA, $150 \mathrm{mM} \mathrm{KCl}$, and $0.085 \mathrm{mM}$ PyMIn (open circles) or $0.053 \mathrm{mM}$ PyMInMI (filled circles). The residual activity is given in percent of the activity at $t=0$ on a logarithmic scale. The curved lines are fitted as for Fig. $2 \mathrm{~A}$, yielding second-order rate constants for the two phases of inactivation of 3.8 and $0.28 \mathrm{mM}^{-1} \cdot \mathrm{min}^{-1}$ for PyMIn and 2.1 and $0.16 \mathrm{mM}^{-1} \cdot \mathrm{min}^{-1}$ for PyMInMI, respectively. Note that the amplitudes of the fast and slow phases were in proportions 60 and $40 \%$, respectively, in this biphasic fit.

and can be resolved into two components, one with an amplitude of about $80 \%$ of activity which is lost rapidly, and the remaining $20 \%$ having a 40 -fold lower rate of disappearance (Fig. 2A). Note that the incubation medium used in the experiment shown in Fig. $2 \mathrm{~A}$ is lacking ATP, to allow comparison with our previously published NEM inactivation study (18). Rates of inactivation in the presence of ATP are 2- to 10-fold slower than in the absence of ATP.

The time courses of inactivation (in the same incubation medium as for 5-(2)-CBziVSL) by two non-spin-labeled indanediones (PyMIn and PyMInMI) are shown in Fig. 2B. Again biphasic inactivation curves are obtained, with the noncharged label (PyMIn) showing the highest reactivity (see legend to $\mathrm{Fig}$. $2 \mathrm{~B}$ ), with a secondorder rate constant for the rapid phase of 3.8 $\mathrm{mM}^{-1} \cdot \mathrm{min}^{-1}$. The second-order rate constants for inactivation in the presence of ATP are smaller both for the rapid and the slow phase. Values obtained for the two components are 1.7 and $0.11 \mathrm{mM}^{-1} \cdot \mathrm{min}^{-1}$ for PyMIn, and 0.55 and $0.050 \mathrm{mM}^{-1} \cdot \mathrm{min}^{-1}$ for PyMInMI, respectively.

Inactivation (in the presence of ATP) by each of the 14 labels shown in Fig. 1 was studied with incubation times at $37^{\circ} \mathrm{C}$ up to $2 \mathrm{~h}$. Most of the labels had a rather low reactivity, so the biphasic behaviour seen in Fig. $2 \mathrm{~A}$ was not always evident within the 2 -h incubation period.
The initial part of the inactivation curve yielded the rate constants given in Table 1 for the various labels. Most of the labels were insoluble in the incubation medium above a concentration of $2 \mathrm{mM}$. This limited the extent of inactivation for the slowly reacting labels to about $70 \%$ residual activity after $2 \mathrm{~h}$ (see Table 1 ). In the following, we have used $1-\mathrm{h}$ incubation periods for labeling of the Na,K-ATPase as a standard procedure since we have previously observed a temperature-dependent (and time-dependent) aggregation of the protein $(9,14)$.

The conventional ESR spectra recorded at $0^{\circ} \mathrm{C}$ of $\mathrm{Na}, \mathrm{K}$-ATPase labeled with the 12 different spin-labeled derivatives are given in Fig. 3. The 5-(3)-IndVSL label is excluded because of its very low reactivity. The major portion of the spin-label is strongly immobilized on the conventional ESR time scale for all 12 spectra, although a sharp mobile component is present for some of the labels. All spectra have a degree of immobilization comparable to that obtained with 5-BzVSL-labeled Na,K-ATPase (9).

The second harmonic, $90^{\circ}$ out-of-phase, absorption STESR spectra $\left(V_{2}^{\prime}\right)$ of $\mathrm{Na}, \mathrm{K}$-ATPase labeled with the 12 sulfhydryl reagents are given in Fig. 4. The spectra are minimally distorted by the small mobile spin label component, and the lineheight ratios in the diagnostic STESR regions (19) can be readily measured (except for 5-EtCxVSL and 5-MeldVSL). Values for the low-, mid-,

TABLE 1

Rates of Inactivation of Na,K-ATPase by Different Covalent Modification Reagents

\begin{tabular}{lllc}
\hline \multicolumn{1}{c}{ SL } & MW & $\begin{array}{c}\text { Conc. } \\
(\mathrm{mM})\end{array}$ & $\begin{array}{c}k_{\mathrm{i}} \\
\left(\mathrm{mM}^{-1} \cdot \mathrm{min}^{-1}\right)\end{array}$ \\
\hline 5-BzVSI ${ }^{a}$ & 268 & 1.00 & 0.0035 \\
5-CBzVSL & 314 & 1.34 & 0.0071 \\
5-HBzVSL & 286 & 1.31 & 0.0036 \\
5-HNBzVSL & 332 & 1.32 & 0.0087 \\
5-HDiNBzVSL & 378 & 1.34 & 0.038 \\
5-(2)-PyVKSL & 440 & 1.32 & 0.0070 \\
5-(3)-IndVSL & 323 & 1.32 & $<0.0001$ \\
5-(2)-CBziVSL & 310 & 0.32 & 0.060 \\
5-DiCVVSL & 216 & 0.46 & 0.017 \\
5-EtCxVSL & 263 & 1.27 & 0.014 \\
5-DiBzVSL & 374 & 1.32 & 0.0025 \\
5-InVSL & 296 & 0.078 & 0.68 \\
6-InVSL & 310 & 0.65 & 0.026 \\
5-MeldVSL & 294 & 1.13 & 0.0027 \\
\hline
\end{tabular}

Note. $\mathrm{Na}, \mathrm{K}$-ATPase was incubated at $37^{\circ} \mathrm{C}$ in the presence of 30 mM histidine ( $\mathrm{pH} 7.4$ at $37^{\circ} \mathrm{C}$ ), $5 \mathrm{~mm}$ CDTA, $150 \mathrm{~mm} \mathrm{KCl,} 3 \mathrm{mM}$ ATP and spin label reagent at the concentration indicated. The loss of activity was followed for $2 h$. The inactivation rate constants are measured from the initial phase of inactivation. All inactivation curves were clearly biphasic (cf. Fig. 2) when more than $50 \%$ inactivation was achieved.

${ }^{\circ}$ Data from Ref. (9). 


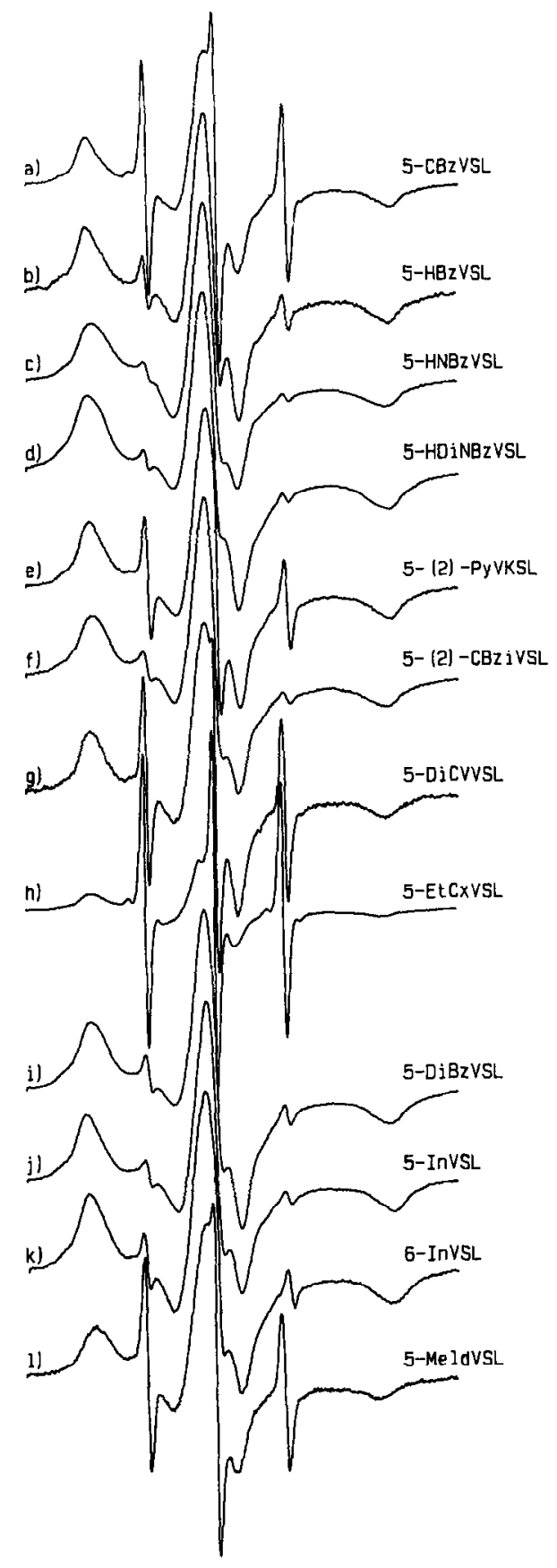

FIG. 3. Conventional ESR spectra $\left(V_{1}\right)$, at $0^{\circ} \mathrm{C}$, of Na,K-ATPase membranes labeled at Class II groups with various nitroxide derivatives. The spectra shown are for enzyme labeled with (a) 5-CBzVSL, (b) 5-HBzVSL, (c) 5-HNBzVSL, (d) 5-HDiNBzVSL, (e) 5-(2)PyVKSL, (f) 5-(2)-CBziVSL, (g) 5-DiCVVSL, (h) 5-EtCxVSL, (i) 5DiBzVSL, (i) 5-InVSL, (k) 6-InVSL, and (1) 5-MeldVSL, respectively. Total scan width, $100 \mathrm{G}$.

and high-field lineheight ratios are given in Table 2, as well as the values for the normalized integrals. The effective rotational correlation times deduced from the lineheight ratios and normalized integrals, using calibrations from isotropically tumbling spin-labeled hemoglobin, are within the range observed with 5-BzVSL (9).
The most reactive of these new spin-labels is 5InVSL. A detailed kinetic analysis yields rate constants of inactivation for the fast and slow phases (determined as shown for 5-(2)-CBziVSL in Fig. 2) for 5-InVSL of 3 and $0.1 \mathrm{mM}^{-1} \cdot \mathrm{min}^{-1}$, respectively. These values are measured in the absence of ATP and can thus be compared to our previous estimates of inactivation with NEM (18), where we obtained values of 0.5 and 0.05 $\mathrm{mM}^{-1} \cdot \mathrm{min}^{-1}$, respectively (at $0.1 \mathrm{mM} \mathrm{NEM}$ ). 5 -InVSL is thus more reactive than the maleimide spin-label and gives a more homogeneous ESR spectrum, i.e., is much better suited for STESR investigations.

Thus all the labels have the same spectral characteristics, indicative of the protein rotational dynamics, even though the reaction rates for the labels vary widely.

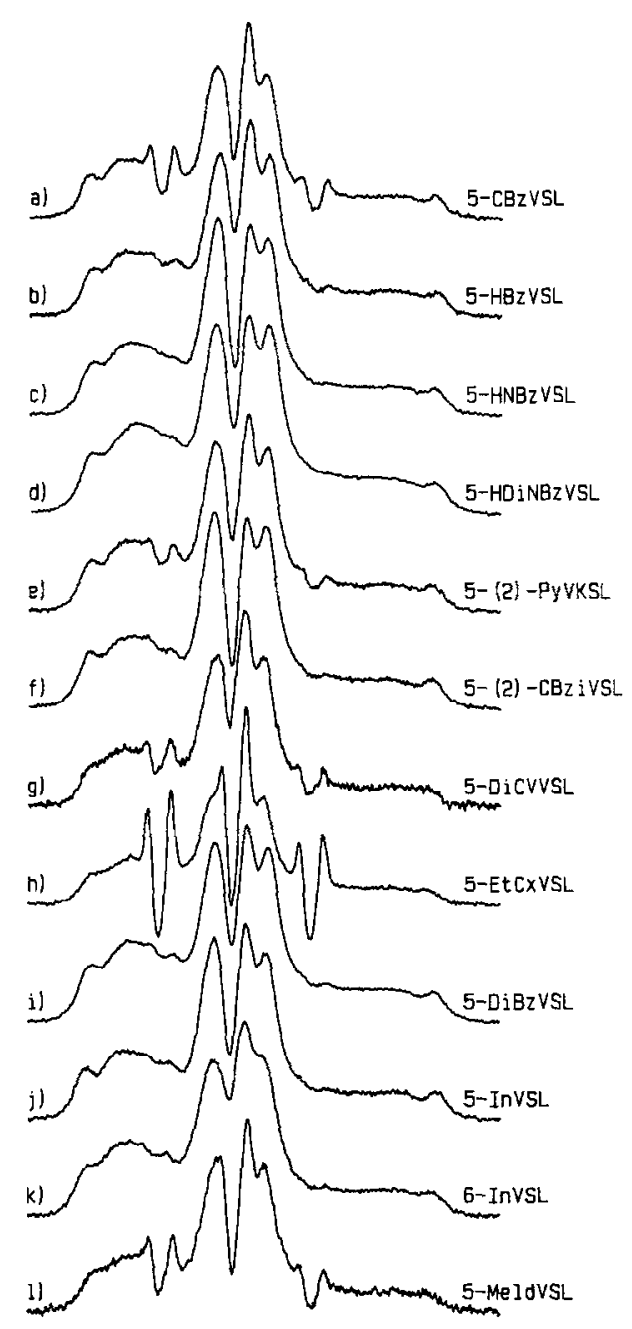

FIG. 4. Second harmonic, $90^{\circ}$ out-of-phase STESR spectra $\left(V_{2}^{\prime}\right)$ at $0^{\circ} \mathrm{C}$ of $\mathrm{Na}, \mathrm{K}-\mathrm{ATP}$ ase labeled at Class II groups. The spectra shown are for enzyme labeled with (a) 5-CBzVSL, (b) 5-HBzVSL, (c) 5HNBzVSL, (d) 5-HDiNBzVSL, (e) 5-(2)-PyVKSL, (f) 5-(2)CBziVSL, (g) 5-DiCVVSL, (h) 5-EtCxVSL, (i) 5-DiBzVSL, (j) 5InVSL, (k) 6-InVSL, and (l) 5-MeldVSL, respectively. Total scan width, $100 \mathrm{G}$. 
TABLE 2

STESR Parameters at $0^{\circ} \mathrm{C}$ for $\mathrm{Na}, \mathrm{K}$-ATPase Labeled at Class II SH Groups

\begin{tabular}{lccll}
\hline \multicolumn{1}{c}{ SL } & \multicolumn{1}{c}{$L^{\prime \prime} / L$} & $C^{\prime} / C$ & \multicolumn{1}{c}{$H^{\prime \prime} / H$} & $I_{\mathrm{ST}} \times 10^{2}$ \\
\hline 5-CBzVSL & $1.37(330)$ & 0.39 & $0.92(90)$ & $0.71(80)$ \\
5-HBzVSL & $1.37(320)$ & 0.51 & $1.06(140)$ & $0.85(165)$ \\
5-HNBzVSL & $1.37(330)$ & 0.24 & $1.05(140)$ & $0.51(25)$ \\
5-HDiNBzVSL & $1.44(440)$ & 0.45 & $1.19(220)$ & $0.53(25)$ \\
5-(2)-PyVKSL & $1.37(330)$ & 0.41 & $1.05(140)$ & $0.64(50)$ \\
5-(2)-CBziVSL & $1.25(190)$ & 0.21 & $1.01(120)$ & $0.45(15)$ \\
5-DiCVVSL & $1.33(280)$ & 0.53 & $1.0(120)$ & $0.71(75)$ \\
5-EtCxVSL & $1.45(470)$ & -0.01 & $1.08(150)$ & $0.75(95)$ \\
5-DiBzVSL & $1.39(360)$ & 0.43 & $1.09(160)$ & $0.57(35)$ \\
5-InVSL & $1.35(300)$ & 0.35 & $0.96(100)$ & $0.71(75)$ \\
6-InVSL & $1.46(490)$ & 0.76 & $1.10(170)$ & $0.76(100)$ \\
5-MeldVSL & & & \\
\hline
\end{tabular}

Note. Values for the low-field $\left(L^{\prime \prime} / L\right)$, the midfield $\left(C^{\prime} / C\right)$, and highfield $\left(H^{\prime \prime} / H\right)$ lineheight ratios are obtained from the STESR spectra shown in Fig. 4. The normalized integrals $\left(I_{\mathrm{ST}}\right)$ are measured as described by Horváth and Marsh (17). Numbers in parentheses give the rotational correlation times (in $\mu \mathrm{s}$ ) deduced from calibrations of the spectral parameters (23).

a Values are possibly affected by the presence of an overlapping sharp spectral component. For 5-DiCVVSL, 5-CBzVSL, and 5-(2)PyVKSL this is only likely to affect the central region $\left(C^{\prime} / C\right)$.

The temperature dependence of the STESR spectra of Na,K-ATPase labeled at Class II SH groups with 5InVSL is illustrated in Fig. 5. The spectra indicate an increasing degree of rotational mobility with increasing temperature, as expected. However, an irreversible decrease in mobility is observed on returning to $0^{\circ} \mathrm{C}$ after cycling to $37^{\circ} \mathrm{C}$ (compare the upper and lower spectra in Fig. 5). The values for $H^{\prime \prime} / H$ and $I_{\mathrm{ST}}$ are at $0^{\circ} \mathrm{C}$ increased from 0.96 and 0.71 , respectively, to 1.02 and 0.82 , respectively, after cycling to $37^{\circ} \mathrm{C}$.

\section{DISCUSSION}

The present paper describes covalent modification of the $\mathrm{Na}, \mathrm{K}$-ATPase with a series of cysteine-reactive nitroxides. There are two important issues to be discussed, namely, the spectroscopic characteristics of the labeled protein (conventional ESR as well as STESR) and the reactivity of the sulfhydryl reagents -and thus implications for protein modification.

\section{Spectroscopic Characteristics}

Conventional ESR. All the spectra obtained here are multicomponent spectra, with most of the spin label intensity residing in the component corresponding to immobilized spin labels. Some of the labels give rise to a sharp, mobile component, which may distort the STESR spectra (see below), notably 5-CBzVSL, 5EtCxVSL, and 5-MeldVSL. For other labels, e.g., 5-(2)-
CBziVSL, 5-HNBzVSL, 5-DiBzVSL, and 5-InVSL, the mobile component is negligible.

The shape of the immobilized component also reveals heterogeneity of labeling in certain cases-compare, for example, the spectra of 5-InVSL with 5-(2)-CBziVSL or 5 -HDiNBzVSL. This is consistent with two or more SH groups being labeled, which is also expected, since inactivation (and thus labeling) of the $\mathrm{Na}, \mathrm{K}$-ATPase with NEM involves several SH groups (13). Figure 6 shows the relation between degree of inactivation and the nitroxide ESR intensity, the latter being a measure of the spin concentration associated with the labeled protein. There is a fairly direct correlation between nitroxide incorporation into the protein and inactivation. If the labeling time is increased beyond that required for full inactivation, an increase in ESR intensity is to be expected, arising from labeling of sulfhydryl groups that are not important for overall activity (or simply have a low reactivity). With the labels shown in Fig. 6, the conditions for labeling are such that incorporation is into vital sulfhydryl groups. Under these conditions ( $100 \mu \mathrm{M}$ for $60 \mathrm{~min}$ at $37^{\circ} \mathrm{C}$ ), 5-MSL gives an enzyme preparation with about $15 \%$ residual activity and an ESR intensity (in the units Fig. 6) of approximately 80. Assuming that 5-MSL reacts as does NEM, this is equivalent to approximately three groups labeled per subunit, deduced from the incorporation of $\left[{ }^{14} \mathrm{C}\right] \mathrm{NEM}(7)$. This allows a calibration of the spin label intensity in Fig. 6 in terms of numbers of $\mathrm{SH}$ groups labeled. Further clarification of the stoichiometry and site of labeling within the subunit must await the synthesis of radioactive spin labels.

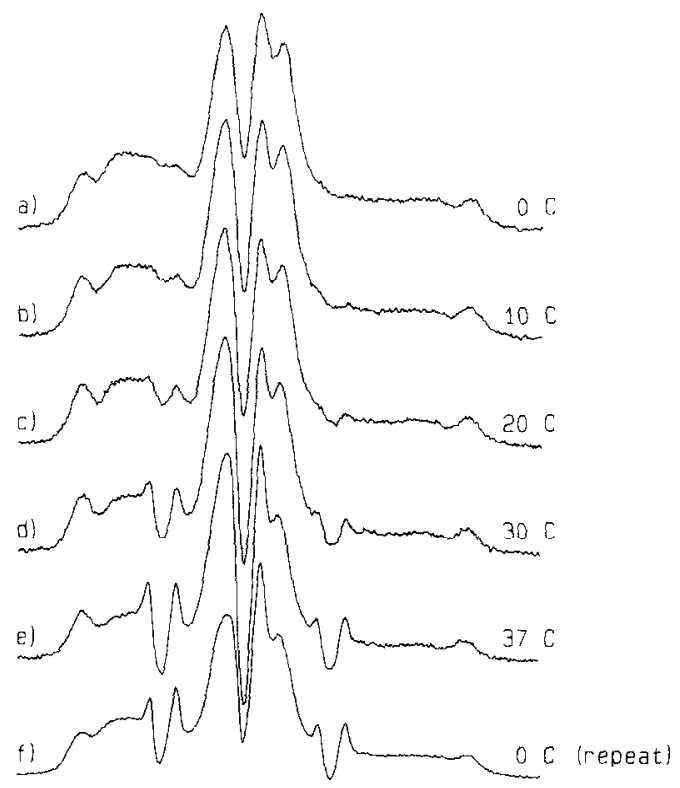

FIG. 5. Temperature dependence of the second harmonic, $90^{\circ}$ outof-phase STESR spectra $\left(V_{2}^{\prime}\right)$ of $\mathrm{Na}, \mathrm{K}$-ATPase labeled on Class II groups with 5 -InVSL. The bottommost spectrum was recorded at $0^{\circ} \mathrm{C}$ after spectra from 0 to $37^{\circ} \mathrm{C}$ were recorded. Total scan width, $100 \mathrm{G}$. 


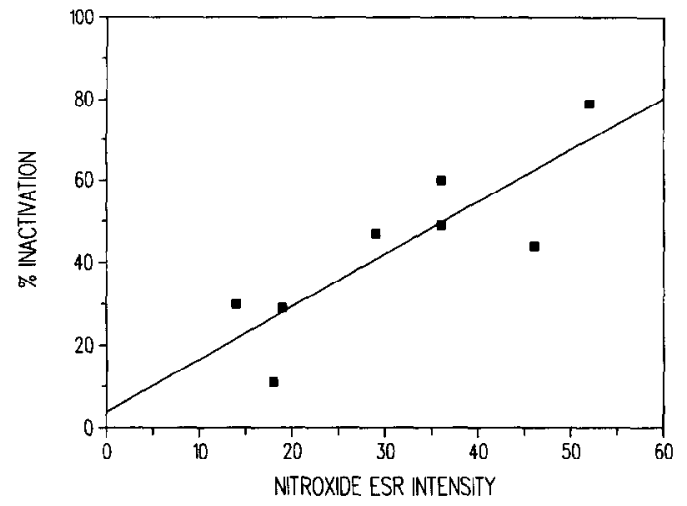

FIG. 6. Correlation between the degree of inactivation by the various spin labels and the incorporation of spin label, measured as the double integral (arbitrary units) of the conventional ESR spectrum. The line shows a linear regression of the data. Four labels (5HNBzVSL, 5-HDiNBzVSL, 5-(2)-CBziVSL, and 5-DiBzVSL) were not included in this correlation due to the presence of two components in the peak related to strongly immobilized spin labels (cf. Fig. 3 and text).

The labels that give rise to two-component spectra which are not included in Fig. 6 have nitroxide ESR intensities considerably higher than would be predicted from the correlation with degree of inactivation given in the figure. This indicates that the second ESR component for these nitroxide derivatives corresponds to labeled groups not essential for activity. Comparison with the single-component spectra from the other group of labels, together with spectral subtraction, can be used, in principle, to isolate the spectra from this second set of labeling sites. In this way, the different labeling specificities of the various nitroxide reagents could be exploited.

The hyperfine splittings and the linewidths at halfheight for those of the labels which display a homogeneous immobilized component (cf. Fig. 3) can be used to estimate rotational motion in the slow-motional regime of conventional ESR spectroscopy $\left(\tau_{\mathrm{r}}<0.1 \mu \mathrm{s}\right)$. However, the outer hyperfine splittings $\left(2 A_{\max } \sim 70.6 \mathrm{G}\right)$ are unusually large and presumably close to the rigid limit, which limits the sensitivity to which the rotational correlation times can be determined from measurements of $2 A_{\max }$. Calculations from the linewidths (using rigid limit values of 2.03 and $2.15 \mathrm{G}$ for the low- and high-field lines (22)) yield values in the range 10-30 ns, suggesting some limited segmental motion of the labels.

Saturation Transfer ESR. STESR spectra of covalently labeled $\mathrm{Na}, \mathrm{K}$-ATPase reveal slow rotational motion of the protein, with rotational correlation times in the 100 - to $300-\mu$ s time range for all the different labels, irrespective of degree of labeling or reactivity. This range of correlation times is indicative of an oligomeric structure, an $(\alpha \beta)_{2}$ or higher oligomer of the $\mathrm{Na}, \mathrm{K}-\mathrm{ATPase}$ (see Ref. (9) for a discussion).
Our earlier STESR study with 5-MSL was complicated both by multiple labeling and by segmental motion of the attached labels (14). The large difference obtained between the effective rotational correlation times deduced from the saturation transfer intensities and the diagnostic STESR lineheight ratios was attributed to the effects of this segmental motion $(9,14)$. The correlation times deduced from the lineheight ratios and from the normalized integral intensities are in much better agreement for the present set of spin labels (cf. Table 2) than for the maleimide-labeled $\mathrm{Na}, \mathrm{K}-\mathrm{ATP}$ ase (14). This can be interpreted as an absence of extensive segmental motion when the protein is labeled with these new labels, compared to the more complex results obtained with 5 -MSL. The origin of this improvement of labeling lies in the shorter linkage between the nitroxide and the point of attachment, and possibly also the more bulky substituents in the new labels.

The contribution from mobile spin labels is small in protein labeled with the very reactive 5 -InVSL (cf. Fig. 3) and also in labels with bulky substituents such as 5 -DiBzVSL. These labels are therefore well suited for studies of rotational dynamics, giving more easily analyzed spectra than in previous studies with 5-MSL.

In brief, the new nitroxide-labeling reagents such as 5 -InVSL combine high reactivity with improved STESR spectroscopic properties, whereas earlier labels had either high reactivity but complex ESR (5-MSL; Ref. (14)) or readily interpretable ESR spectra but low reactivity (5-BzVSL; Ref. (9)). These properties are a direct result of the design of the labels, whereby the reactive carbon-carbon double bond is placed closer to the nitroxide moiety in the vinyl ketone than in the maleimide labels, and the reactivity is enhanced by appropriate choice of the EWG substituent (cf. Fig. 7). Of the new labels, the indanedione derivative (5-InVSL) appears to be the label of choice for STESR studies, in view of its high reactivity, the absence of segmental motion, and the selectivity of labeling as evidenced by the single-component conventional ESR spectrum. This particular label has very recently been shown also to be highly suitable for STESR studies of the Ca-ATPase (25), hence indicating some generality in the conclusions drawn with the membrane protein studied here.

\section{Reactivity of the Vinyl Ketone Labels}

The rate of reaction between the $\mathrm{SH}$ group of cysteine and carbon-carbon double bond, a nucleophilic attack, depends mainly on the presence of groups that diminish the electron density in the double bond. For example, the double bond of maleimide reagents exhibits a remarkable electrophilicity toward nucleophiles ( $\mathrm{SH}$ groups or amino groups) due to the presence of the two 


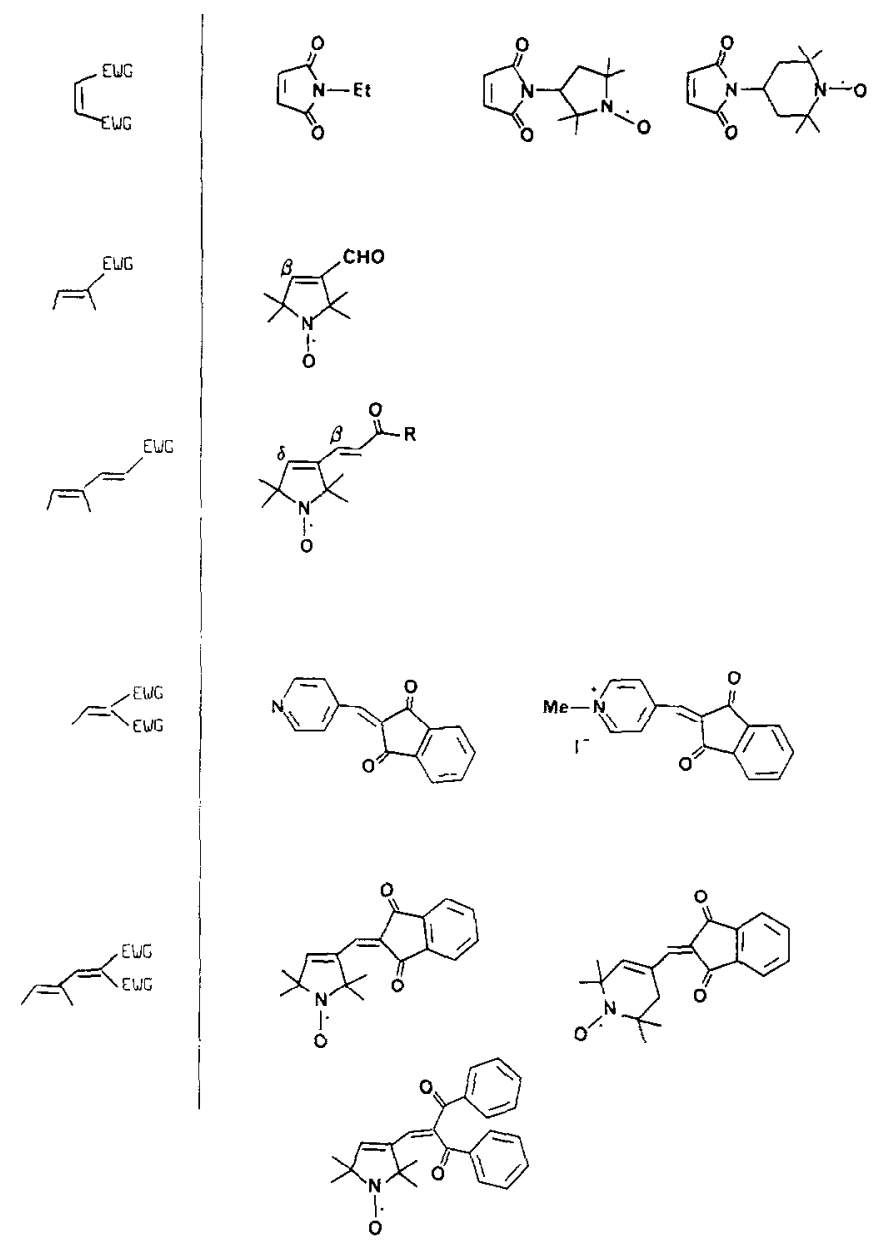

FIG. 7. Principal structural features of the labels used in this paper, classified according to the EWG (electron-withdrawing group) substituents. The first row gives the maleimide derivatives, NEM, 5-MSL, and 6-MSL; the second row represents a simple aldehyde spin label; the third row indicates a generic vinyl ketone derivative; the fourth row gives the PyMIn and PyMInMI labels; and the bottom rows give the 5-InVSL, 6-InVSL, and 5-DiBzVSL labels.

carbonyl electron-withdrawing groups (see Fig. 7, first row). The reactivity of an $\alpha, \beta$-unsaturated ketone or aldehyde is lower, principally because the double bond is polarized by only one EWG. The reactivity may additionally be diminished by the presence of substituents in the vicinity of the electrophilic centre (the double bond is actually an integral part of a bulky pyrroline heterocycle). This is the case for 1-oxyl-3-formyl-2,2,5,5-tetramethyl-3-pyrroline (Fig. 7, second row). When this aldehyde is used in an aldol condensation to form an $\alpha, \beta, \gamma, \delta$-dienone (10) there is the possibility of conjugate addition to either the $\beta$ - or the $\delta$-carbon, according to previous studies on polyunsaturated ketones (21).

However, it was demonstrated that in the reaction of a phenyl dieno ketone (20) with phenylmethanethiol only $\beta$-addition takes place (10). The reactivity of the $\beta$-carbon can be enhanced by the introduction either of a stronger EWG substituent at the $\alpha$-carbon, or of a second EWG at the $\alpha$-carbon (Fig. 7, fourth row). This principle has been used to design this series of new sulfhydryl reagents with different reactivity, offering an opportunity to choose the particular reagent which reacts in the most selective manner.

With the benzoyl-substituted spin labels, a progressive increase in reactivity is observed as the degree of substitution by electrophilic groups is increased; the reactivity is found to be in the order $5-\mathrm{HBzVSL}<5$ $\mathrm{CBzVSL}<5$-HNBzVSL $<$ 5-DiNBzVSL. This corresponds to the increasing electron-withdrawing ability of these substituents.

It is clear, however, that steric properties of the labels also play a role in the reactivity: changing the nitroxide group from a five-membered ring to a six-membered ring (5-InVSL vs 6 -InVSL) decreases reactivity by a factor of 25 (cf. Table 1). The reduced reactivity of the dibenzoyl derivative, relative to the monobenzoyl derivative, most probably occurs because one of the benzoyl substituents is forced into a nonplanar configuration for steric reasons, hence reducing its effectiveness as an EWG. Such nonplanar configurations also cannot be excluded for some of the mono derivatives.

We believe that the above-mentioned principles may form the basis for designing new custom-made spin labels, and that similar principles also can be used to design other highly reactive and water-soluble non-spinlabeled SH reagents, as is demonstrated in the case of 2-(4-pyridyl)indane-1,3-dione and its methiodide salt (Fig. 7, fourth row; cf. also Fig. 2B).

\section{ACKNOWLEDGMENTS}

The authors thank Ms. Angielina Tepper for excellent technical assistance. The synthesis of labels (H.O.H. and K.H.) was supported by grants from the Hungarian Academy of Sciences (301/A/82 and 3/104/86). Financial support to Mikael Esmann was obtained from the Danish Medical Research Council (Grant 12-7444) and from the Danish Biomembrane Research Center.

\section{REFERENCES}

1. Lundblad, R. L., and Noyes, C. M. (1984) Chemical Reagents for Protein Modification, Vol. 1, p. 55 and references cited therein, CRC Press, Boca Raton, FL.

2. Hyde, J. S., and Thomas, D. D. (1980) Annu. Rev. Phys. Chem. 31, 293-317.

3. Thomas, D. D. (1985) in The Enzymes of Biological Membranes (Martonosi, A. N., Ed.) 2nd ed., Vol. 1, pp. 287-312, Plenum, New York.

4. Patai, S., and Rappaport, Z. (1964) in The Chemistry of Functional Groups: The Chemistry of Alkenes (Patai, S., Ed.), Vol. 1, p. 469, Wiley Interscience, New York.

5. Shull, G. E., Schwartz, A., and Lingrel, J. B. (1985) Nature (London) 316, 691-695.

6. Kawakami, K., Noguchi, S., Noda, M., Takahashi, H., Ohta, T., Kawamura, M., Nojima, H., Nagano, K., Hirose, T., Inayama, S., 
Hayashida, H., Miyata, T., and Numa, S. (1985) Nature (London) 316, 733-736.

7. Esmann, M. (1982) Biochim. Biophys. Acta 688, 260-270.

8. Esmann, M., and Klodos, I. (1983) Curr. Top. Membr. Transp. 19, 349-352.

9. Esmann, M., Hankovszky, O. H., Hideg, K., and Marsh, D. (1989) Biochim. Biophys. Acta 978, 209-215.

10. Hankovszky, O. H., Hideg, K., and Jerkovich, G. (1989) Synthesis 526-529.

11. Skou, J. C., and Esmann, M. (1979) Biochim. Biophys. Acta 567, 436-444.

12. Esmann, M. (1988) in Methods in Enzymology (Fleischer, S., and Fleischer, B., Eds.), Vol. 156, pp. 105-115, Academic Press, San Diego, CA.

13. Esmann, M. (1982) Biochim. Biophys. Acta 688, 251-259.

14. Esmann, M., Horváth, L. I., and Marsh, D. (1987) Biochemistry 26, 8675-8683.

15. Fajer, P., and Marsh, D. (1982) J. Magn. Reson. 49, 212-224.
16. Hemminga, M. A., De Jaeger, P. A., Marsh, D., and Fajer, P. (1984) J. Magn. Reson. 59, 160-163.

17. Horváth, L. I., and Marsh, D. (1983) J. Magn. Reson. 54, 363373.

18. Esmann, M., and Nørby, J. G. (1985) Biochim. Biophys. Acta 812, 9-20.

19. Thomas, D. D., Dalton, L. R., and Hyde, J. S. (1976) J. Chem. Phys. 65, 3006-3024.

20. Hankovszky, O. H., Hideg, K., Lex, L., Kulcsár, G., and Halász, H. A. (1982) Canad. J. Chem. 60, 1432-1438.

21. Ingold, C. K. (1969) Structure and Mechanism in Organic Chemistry, 2nd ed., p. 1020, Cornell Univ. Press, Ithaca, NY.

22. Freed, J. (1976) in Spin-Labeling. Theory and Practice (Berliner L. J., Ed.), Vol. 1, pp. 53-132, Academic Press, New York.

23. Horváth, L. I., and Marsh, D. (1988) J. Magn. Reson. 80, 314317.

24. Marsh, D. (1980) Biochemistry 19, 1632-1637.

25. Horváth, L. I., Dux, L., Hankovszky, H. O., Hideg, K., and Marsh, D. (1990) Biophys. J., 58, 231-242. 